BMJ Open Diabetes Research \& Care

\title{
Diabetes mellitus is associated with a higher risk for major depressive disorder in women than in men
}

\author{
Carola Deischinger, ${ }^{1}$ Elma Dervic, ${ }^{2,3}$ Michael Leutner, ${ }^{1}$ Lana Kosi-Trebotic, ${ }^{1}$ \\ Peter Klimek, ${ }^{2,3}$ Alexander Kautzky, ${ }^{4}$ Alexandra Kautzky-Willer (D) ${ }^{1,5}$
}

To cite: Deischinger C, Dervic $\mathrm{E}$, Leutner $\mathrm{M}$, et al. Diabetes mellitus is associated with a higher risk for major depressive disorder in women than in men. BMJ Open Diab Res Care 2020;8:e001430. doi:10.1136/ bmjdrc-2020-001430

- Additional material is published online only. To view please visit the journal online (http://dx.doi.org/10.1136/ bmjdrc-2020-001430).

$\mathrm{CD}$ and $\mathrm{ED}$ are joint first authors.

Received 2 April 2020 Revised 4 August 2020 Accepted 13 August 2020

\section{Check for updates}

(C) Author(s) (or their employer(s)) 2020. Re-use permitted under CC BY-NC. No commercial re-use. See rights and permissions. Published by BMJ.

For numbered affiliations see end of article.

Correspondence to Dr Alexandra Kautzky-Willer; alexandra.kautzky-willer@ meduniwien.ac.at

\section{ABSTRACT}

Introduction Both diabetes mellitus and being female significantly increase the risk of being diagnosed with major depressive disorder (MDD). The diagnosis of MDD, combined with diabetes mellitus, can be detrimental in terms of mortality and morbidity. We aimed at investigating the impact of diabetes mellitus on the gender gap in MDD over the course of a human lifetime.

Research design and methods In a cross-sectional study over the course of 17 years, medical claims data of the general Austrian population ( $\mathrm{n}=8996916$ ) between 1997 and 2014 was analyzed. Of these, 123232 patients with diabetes mellitus were extracted and compared with non-diabetic controls.

Results In a cohort of 123232 patients with diabetes mellitus and 1933218 controls (52\% females, $48 \%$ males), women with diabetes had 2.55 times increased ORs to be diagnosed with MDD compared with women without diabetes $(95 \% \mathrm{Cl} 2.48$ to $2.62, \mathrm{p}<0.001)$ between the age of 30 and 69 years. The effect of diabetes mellitus on the prevalence of MDD was significantly smaller in men $(0 R=1.85,95 \% \mathrm{Cl} 1.80$ to $1.91, p<0.001)$. Between 0 and 30 years and after age 70 years, the gender gap of MDD was not different between patients with and without diabetes mellitus. The peak of the gender gap in MDD in patients with diabetes mellitus was around the age of 40-49 years. A sensitivity analysis identified overweight, obesity and alcohol dependence as the most potent influencing factors of the widening of the gender gap among patients with diabetes mellitus.

Conclusions Diabetes mellitus is a stronger risk factor for MDD in women than in men, with the greatest width of the gender gap between 40 and 49 years. High-risk patients for MDD, such as overweight female patients with diabetes, should be more carefully assessed and monitored.

\section{INTRODUCTION}

Depression and diabetes mellitus are two detrimental burdens on global health, currently affecting 5\% and 9\% of the world's population, respectively. ${ }^{1}$ The prevalence of diabetes mellitus is slightly higher in men than in women and increasing across all age groups. ${ }^{23}$ With one in four type 2 diabetes patients suffering from depression, the probability of being diagnosed is five times

\section{Significance of this study}

What is already known about this subject?

- Type 2 diabetes mellitus patients have an increased risk of being diagnosed with major depressive disorder (MDD), and patients suffering from MDD are at an elevated risk to develop type 2 diabetes mellitus.

- Women are twice as likely to be diagnosed with depression compared with men resulting in a steep gender gap.

What are the new findings?

- Women with diabetes had over two times higher ORs to be diagnosed with MDD compared with women without diabetes $(\mathrm{OR}=2.55,95 \% \mathrm{Cl} 2.48$ to 2.62 , $\mathrm{p}<0.001$ ) between the age of 30 and 69 years. In men with diabetes, the effect was significantly less pronounced ( $0 \mathrm{R}=1.85,95 \% \mathrm{Cl} 1.80$ to $1.91, \mathrm{p}<0.001$ ).

- Overweight, obesity and alcohol dependence were identified as the strongest influencing factors for the widening of the gender gap in MDD among patients with diabetes.

How might these results change the focus of research or clinical practice?

- This is the first time showing that diabetes mellitus is an even greater risk factor for MDD in women than in men.

- High-risk patients, such as overweight female diabetic patients with or without cardiovascular disease, should be even more carefully assessed and monitored concerning their risk for MDD.

- Females with diabetes had over two times higher ORs to be diagnosed with MDD compared with women without diabetes between the age of 30 and 69 years. In men with diabetes, the effect was significantly less pronounced.

- Overweight, obesity and alcohol dependence were identified as the strongest influencing factors for the widening of the gender gap in MDD among patients with diabetes.

- The gender gap in MDD is more substantial among patients with diabetes mellitus compared with nondiabetic controls.

higher than in the non-diabetic population. ${ }^{45}$ Diabetes mellitus and depression appear to display a certain etiological bidirectionality 
as also patients with depression show an elevated risk to develop type 2 diabetes mellitus. ${ }^{6}$ In combination, these two chronic conditions result in 4.5 times higher healthcare costs than for diabetes patients without depression ${ }^{7}$ due to both diseases being associated with an increase in mortality and morbidity. ${ }^{89}$

Type 2 diabetes mellitus is a chronic, non-communicable disease with serious long-term complications such as cardiovascular, peripheral vascular, ocular, neurologic, renal and reproductive abnormalities. Depression worsens the prognosis in patients with diabetes by increasing the rate of diabetic complications as well as the risk of cardiac mortality. ${ }^{81011}$

Depression affects mortality and morbidity negatively in both sexes. ${ }^{8}$ In female patients with diabetes, however, it results in a 2-3 times higher risk for cardiovascular diseases compared with women without diabetes. ${ }^{3}{ }^{12}$ The postmenopausal decrease in the seemingly cardioprotective estrogen ${ }^{13}$ and the perimenopausal peak of depression may explain this more pronounced effect in patients with diabetes. ${ }^{14}$ With women being twice as likely to be diagnosed with depression than men, there is a steep gender gap in the diagnosis of depression, which might be influenced by an interaction of diabetes mellitus with the diagnosis of depression. ${ }^{451516}$ Reflecting on these previous findings, we investigated the effect of diabetes mellitus on the gender gap in major depressive disorder (MDD) as well as incidence rates for depression over the course of a lifetime.

\section{STUDY DESIGN AND METHODS}

A cross-sectional study of a medical claims database spanning 17 years of data from the Austrian Ministry of Health was conducted. The database contained records of all hospitalized Austrians between 1997 and 2014 (n=8 996 916), a total number of 45000000 hospital stays. Each record consists of a unique patient and stay identifier, the patient's age group, sex, primary diagnose, secondary diagnoses as well as hospital entry and exit dates. The database includes 1699 disorders classified with threedigit International Classification of Diseases, 10th Revision (ICD-10) codes. In Austria, for each hospital stay, a primary diagnosis is determined and a varying number of secondary diagnoses might be added. To ensure the comparability of health status of our study population, we restricted the analysis to patients without any hospital encounters from 1997 to 2002 and at least one hospital stay between 2003 and 2014. Furthermore, the ICD coding system changed to ICD-10 2001 in Austria in the early 2000s; mixing different classification systems would have rendered the analysis unreliable. We proceeded to select diabetes mellitus patients and an appropriate control group. We defined all patients with at least one diagnosis from the range E10-E14 in the years 2003-2014 (main or side diagnosis) as patients with diabetes and the rest of the population as controls. Patients with depression were identified with the ICD-10 code of F32 or F33 (main or side diagnosis). All patients were categorized into 10-year age groups starting with age 10-19, 20-29, up to 70-79 years. For each age group, we calculated ORs for being diagnosed with MDD in patients with diabetes mellitus and the control group. A similar approach was used to calculate ORs for diagnoses that potentially promote depression. The gender gap was calculated as the ratio between these ORs for women and men. A series of sensitivity analyses was conducted to assess the robustness and potential confounders of our results by excluding patients with diagnoses potentially promoting depression (overweight and obesity (ICD-10 code E66), nicotine dependence (F17) and alcohol-related disorders (F10), renal failure (N17 and N18), cardiovascular disorders (I20-I25), stroke (I63 and I64), atherosclerosis (I70), peripheral artery diseases (I73), hypothyroidism (E02 and E03), cancer (C00-C97 and D00-D48), sleep disorders (G47) and post-traumatic stress disorder (PTSD) (F43)). By excluding these patients, we were able to determine whether the observed relations between depression and diabetes are confounded by any of these diagnoses.

\section{RESULTS}

\section{Baseline characteristics}

We focused on the age groups 30-69 years, with a total of 123232 patients with diabetes (43269 women, 79963 men) and 1933218 controls because there was no significant difference in the gender gap between the diabetes mellitus group and the general population before age 30 years and after 69 years. As visible in table 1, 10.54\% of all patients with diabetes had been diagnosed with MDD ( $15.63 \%$ of women, $7.79 \%$ of men). The control group (no diabetes mellitus) consisted of 1933218 patients (970625 women, 962593 men), $5.41 \%$ of which had received the diagnosis MDD $(6.53 \%$ of women, $4.28 \%$ of men). Baseline characteristics of the population $<30$ years can be found in the online supplemental S1 and S2. Patients with diabetes had more hospital days, stays, a higher total number of diagnoses and were more likely to be diagnosed with obesity and overweight, nicotine dependence, alcohol-related disorders, sleep disorders, MDD, cardiovascular disease, cerebral infarction, diseases of arteries, arterioles and capillaries, kidney failure, hypothyroidism and cancer when compared with the non-diabetic control group.

\section{Sex-specific baseline characteristics}

Female patients with diabetes were older and had on average slightly more diagnoses and hospital days than their male counterparts. The most frequent diagnoses for female and male patients with diabetes overlapped for the most part (E11 - type 2 diabetes mellitus, I10 hypertension, E78 - lipoprotein metabolism disorder, E14 - unspecified diabetes mellitus), except for urinary tract infection (N39) in women and chronic ischemic heart disease (I25) in men. 
Table 1 Baseline characteristics and prevalence (in \%) of major depressive disorder and possible confounding factors for depression among all patients between the age of 30 years and 69 years with and without diabetes mellitus in Austria from 2003 to 2014

\begin{tabular}{|c|c|c|c|c|c|c|}
\hline \multirow[b]{2}{*}{ Parameters and diagnoses } & \multicolumn{3}{|c|}{ With diabetes } & \multicolumn{3}{|c|}{ Without diabetes } \\
\hline & All & Female & Male & All & Female & Male \\
\hline Age (in years) & $57.53 \pm 9.22$ & $57.7 \pm 9.53$ & $57.44 \pm 9.04$ & $48.65 \pm 10.87$ & $48.18 \pm 10.89$ & $49.13 \pm 10.83$ \\
\hline Hospital stays & $5.89 \pm 8.31$ & $5.89 \pm 8.26$ & $5.89 \pm 8.34$ & $2.85 \pm 4.53$ & $2.88 \pm 4.63$ & $2.82 \pm 4.43$ \\
\hline Hospital days (in days) & $47.52 \pm 75.17$ & $47.85 \pm 73.24$ & $47.33 \pm 76.19$ & $17.07 \pm 52.39$ & $16.15 \pm 51.43$ & $17.99 \pm 53.32$ \\
\hline Number of hospital diagnoses & $10.22 \pm 7.72$ & $10.27 \pm 7.73$ & $10.2 \pm 7.7$ & $3.88 \pm 4.03$ & $3.8 \pm 3.93$ & $3.96 \pm 4.13$ \\
\hline All patients & 123232 & 43269 & 79963 & 1933218 & 970625 & 962593 \\
\hline E66 - obesity and overweight (in \%) & 26.41 & 31.07 & 23.89 & 4.33 & 4.36 & 4.30 \\
\hline F17 - nicotine dependence (in \%) & 12.33 & 8.59 & 14.36 & 4.63 & 3.13 & 6.13 \\
\hline F10 - alcohol-related disorders (in \%) & 5.79 & 2.57 & 7.54 & 3.88 & 1.90 & 5.88 \\
\hline $\begin{array}{l}\text { G47 - sleep disorders } \\
\text { (in \%) }\end{array}$ & 6.38 & 3.88 & 7.74 & 2.38 & 1.10 & 3.67 \\
\hline $\begin{array}{l}\text { F32-F33 - depression } \\
\text { (in \%) }\end{array}$ & 10.54 & 15.63 & 7.79 & 5.41 & 6.53 & 4.28 \\
\hline |20-|25 - cardiovascular diseases (in \%) & 24.37 & 17.29 & 28.21 & 4.9 & 2.53 & 6.66 \\
\hline 163-I64 - cerebral infarction (in \%) & 6.44 & 5.14 & 7.15 & 1.28 & 0.90 & 1.66 \\
\hline $\begin{array}{l}\text { 170-179 - diseases of arteries, arterioles } \\
\text { and capillaries (in \%) }\end{array}$ & 6.99 & 5.03 & 8.05 & 0.84 & 0.52 & 1.16 \\
\hline $\begin{array}{l}\text { N17-N19 - acute kidney failure and } \\
\text { chronic kidney disease (in \%) }\end{array}$ & 5.45 & 4.83 & 5.78 & 0.78 & 0.50 & 1.05 \\
\hline E02-E03 - hypothyroidism (in \%) & 5.51 & 9.60 & 3.30 & 2.04 & 3.05 & 1.03 \\
\hline $\begin{array}{l}\text { C00-D48 - neoplasms } \\
\text { (in \%) }\end{array}$ & 1.51 & 2.08 & 1.19 & 1.39 & 1.83 & 0.95 \\
\hline
\end{tabular}

\section{Sex-specific comparison of the diagnosis MDD in the general} population over the course of a lifetime

As illustrated in figure 1 , female patients with diabetes displayed a higher risk for developing MDD compared with men with diabetes and the non-diabetic control group in all age groups starting in and around puberty. In the age group of 10-19 years, women (3.07\%) display a higher probability of being diagnosed with MDD than

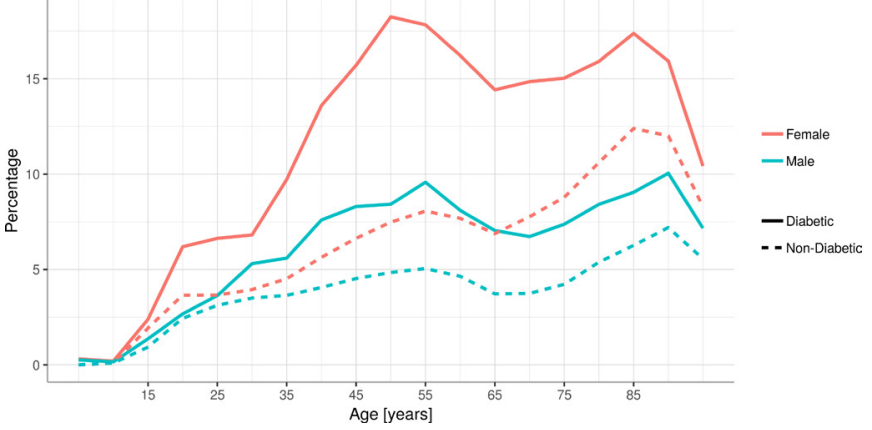

Figure 1 Percentage of patients with diabetes diagnosed with MDD compared with patient without diabetes: gender gap of female and male patients suffering from depression is larger in the diabetes cohort between age 30 and 69 years (female diabetes patients: $\mathrm{OR}=2.55,95 \% \mathrm{Cl} 2.48$ to 2.62 , $\mathrm{p}<0.001$; male diabetes patients: $\mathrm{OR}=1.85,95 \% \mathrm{Cl} 1.80$ to 1.91, $\mathrm{p}<0.001)$. MDD, major depressive disorder. men $(1.84 \%)$. The most prominent gender gap in MDD among patients with diabetes mellitus could be found between the age of 40 and 49 years. Known diagnoses that are potentially promoting depression were compared in patients with diabetes and patients without diabetes. The analysis showed that women suffering from diabetes mellitus versus women without diabetes between the age of 40 and 49 years had the highest ORs of having diseases potentially related to depression such as cardiovascular diseases $($ OR females=5.48; OR males $=4.05)$, overweight and obesity (OR females $=12.37$; OR males $=7.82$ ), stroke (OR females $=4.17$; OR males $=3.55)$, lipoprotein metabolism disorders $(\mathrm{OR}$ females $=8.73$; OR males $=5.65)$, renal failure (OR females=8.80; OR males $=5.84)$ and sleep disorders (OR females=3.31; OR males $=1.97)$, compared with any other age group and compared with men with diabetes (see figure 1 and online supplemental S3 and S4 for details).

\section{Impact of diabetes mellitus on the gender gap in MDD}

In this study, the gender gap in depression is greater in the diabetes cohort than in non-diabetic controls between the age of 30 and 69 years (see figure 1). Here, women with diabetes are at an increased risk to be diagnosed with MDD compared with women without diabetes $(\mathrm{OR}=2.55$, $95 \%$ CI 2.48 to $2.62, \mathrm{p}<0.001$ ), whereas men with diabetes 


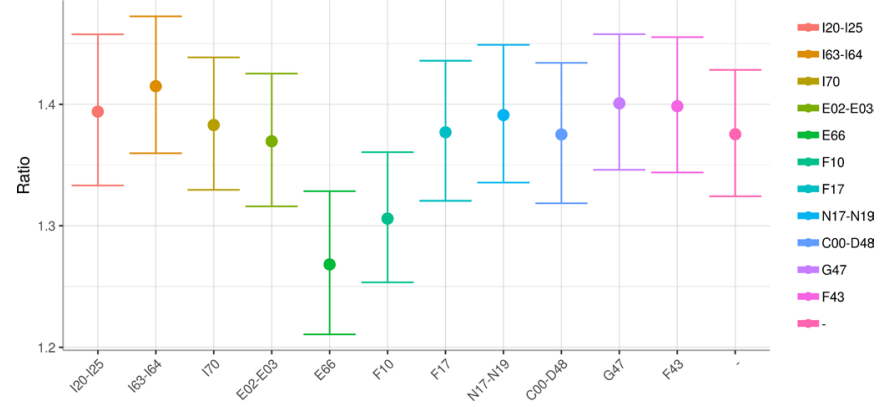

Figure 2 Sensitivity test showing confounding factors for the wider gender gap in MDD among patients with diabetes: obesity (ICD-10 code E66) and alcohol-related disorders (F10) significantly reduced the effect size of diabetes on the gender gap in the diagnosis of depression. Renal failure (N17 and N18), nicotine dependence (F17), cardiovascular diseases (I20-I25), stroke (I63 and I64), atherosclerosis (I70), cancer and PTSD (F43) did not affect the gender gap. ICD10, International Classification of Diseases, 10th Revision; MDD, major depressive disorder; PTSD, post-traumatic stress disorder

only have a 1.85 times higher risk when compared with men without diabetes (95\% CI 1.80 to $1.91, \mathrm{p}<0.001)$, which is significantly lower than in women $(\mathrm{p}<0.0001)$. In other words, women with diabetes showed a gender gap with a 1.37-fold (95\% CI 1.32 to 1.43) increased risk for the diagnosis of depression when compared with male patients with diabetes between the age of 30 and 69 years.

When investigating F32 (MDD, single episode) and F33 (MDD, recurrent) as separate entities, the results did not change significantly (see online supplemental figures S5-S8 in supplementary materials).

To investigate possible confounding factors for the greater gender gap in depression among diabetes patients between 30 and 69 years, we conducted a sensitivity test (figure 2) on diagnoses that potentially influence depression risk. Renal failure (N17 and N18), nicotine dependence (F17), cardiovascular diseases (I20-I25), stroke (I63 and I64), atherosclerosis (I70), cancer and PTSD (F43) did not affect the gender gap. We could identify overweight and obesity (E66) as one of the most important influencing factors for the gender gap. A similar effect could be detected for alcohol-related disorders (F10).

\section{DISCUSSION}

We aimed at investigating the effect of diabetes mellitus on the gender gap in MDD. This is the first study showing that diabetes mellitus is a stronger risk factor for depression in women than in men. The gender gap in the diagnosis of depression is significantly larger in patients with diabetes than in the non-diabetic control group. Furthermore, there is an age peak in the gender gap of MDD at age 40-49 years, which is more pronounced among patients with diabetes than non-diabetic controls.

A gender gap in depression is a well-published finding; ${ }^{15} 16$ however, age-related changes in depression risk and the impact of diabetes have not been sufficiently investigated so far. In the present analysis, women with diabetes mellitus displayed an increased risk to develop depression compared with male patients with diabetes in all age groups. We observed a first rise in MDD around puberty, which was steeper in women than men and, thus, supports previous studies describing the emergence of a gender gap in MDD in this age group. ${ }^{17}{ }^{18}$ Possible explanations are the earlier puberty onset in girls and gender differences in risk factors for depression during childhood combined with environmental stressors. ${ }^{17}{ }^{18} \mathrm{In}$ this analysis, the largest gender gap was found between 40 and 49 years. Correspondingly, women with diabetes mellitus in this age group had a higher OR for diseases that potentially promote depression compared with men. As cardiovascular diseases and obesity, among others, increase the risk for depression, ${ }^{19-21}$ their higher ORs at $40-49$ years in women with diabetes might offer an explanation for the most prominent gender gap in MDD being at the same age. Furthermore, adult women are about two times more likely to be diagnosed with depression, have a higher prevalence of MDD due to biological factors and the psychological burden of the disease and/or men being underdiagnosed with depression. ${ }^{1516}$ A perimenopausal rise in depression rates was demonstrated in longitudinal studies on premenopausal women ${ }^{22} 23$ and associated with hormonal fluctuations rather than absolute hormone values in previous studies. ${ }^{14}{ }^{24}$ Concerning males, research suggests that men are under-reporting depressive symptoms and the severity thereof, ${ }^{25}$ are less likely to seek help ${ }^{16}$ and that the Diagnostic and Statistical Manual of Mental Disorders, fifth revision diagnostic criteria better reflects symptoms of depressed women than men. ${ }^{26}$ Therefore, a case of depression in a male patient is significantly less likely to be recognized by a healthcare professional than one in a female patient, which possibly contributes to the gender gap in depression. ${ }^{25-27}$

In order to investigate potential confounding factors for the wider gender gap among patients with diabetes mellitus, a sensitivity test on known important covariates for depression was conducted. ${ }^{20}{ }^{21}$ 28-32 The sensitivity analysis showed that female patients with diabetes are relatively more affected by overweight, obesity and alcohol-related disorders in the diagnosis of MDD than their male counterparts. Correspondingly, research suggests a strong interrelation between obesity and depression, especially in women, due to physical impairment, social dysfunction, emotional eating, biomolecular changes and both conditions being stigmatizing. ${ }^{19} 2133$ Studies link abdominal obesity in women to an impaired hypothalamic-pituitary-adrenal axis stress response which, in turn, is related to psychiatric disorders such as MDD and anxiety disorders. ${ }^{3}$ However, alcohol-related disorders are typically more prevalent in men and men are more prone to cope with depression via increased alcohol consumption. ${ }^{34} 35$ Some studies, though, have demonstrated a link between alcohol-related disorders and depression in female adolescents, but not 
males. ${ }^{36-38}$ This gender-specific association is believed to decrease with age ${ }^{36}$; however, gender differences in alcohol-related disorders and MDD remain unclear. ${ }^{39}$ Furthermore, literature suggests a similar influence of PTSD on type 2 diabetes mellitus like $\mathrm{MDD}^{40}$; however, PTSD did not significantly impact our results according to the sensitivity analysis. Other potential influencing factors for a wider gender gap in patients with diabetes include women being psychologically more affected by diabetes distress. ${ }^{41-43}$ Furthermore, it has been reported in previous studies that the frequency of doctor visits increases the probability of being diagnosed with depression. ${ }^{44}$ Men are known to visit the doctor less often, possibly resulting in an underdiagnosis of depression in male patients. ${ }^{26-47}$ This effect might be even more prominent in a multimorbid condition such as diabetes mellitus, which requires an increased amount of medical care. Along these lines, we observed that female patients with diabetes have more hospital diagnoses and hospital days than men with diabetes in our cohort.

There are limitations and strengths to this study. We had access to hospital diagnoses of the whole Austrian population from 1997 to 2014, which is a large dataset with a high number of patients. However, due to the character of the dataset, outpatient visits were not recorded. Patients had to have been admitted to a hospital due to diabetes mellitus or any other disease at least once to be included in the analysis. A clear distinction between effects of a single episode (F32) compared with recurring depression (F33) on diabetes mellitus risk is hardly possible in this dataset. Both can only be analyzed as categorical diagnoses due to the cross-sectional nature of the data, which does not account for longitudinal changes or disease progression. Furthermore, data on glycemic control and medication would have opened possibilities to distinguish between patients with poor or good glycemic control and its potential effects on mental health.

In conclusion, diabetes mellitus appears to be a stronger risk factor for the diagnosis of MDD in women than in men. Obesity, overweight and alcohol-related disorders are more potent moderators of MDD risk in female than male diabetes patients. Therefore, we propose that highrisk patients such as overweight female patients with diabetes should be carefully assessed and monitored concerning their individual risk to develop depression.

\section{ETHICS}

We made secondary use of a research database of medical claims records that are safeguarded and maintained by the Main Association of Austrian Social Security Institutions (HVB) and where it has been ensured that no individual is identifiable. This is a consolidated research database that is only available to selected partners under a strict data protection policy. Use of the data takes place in agreement and cooperation with HVB. The data contain no names of individual patients or healthcare providers.
All unique identifiers including postal codes and date of birth have been removed. All team members working with the data have signed a confidentiality agreement and declaration of commitment ensuring that research will be undertaken in accordance with the applicable data protection regulations. A security clearance for this database signed by the legal department of the Medical University of Vienna stating that individuals are identifiable can be provided on request from the corresponding author.

Author affiliations

${ }^{1}$ Department of Medicine III, Endocrinology and Metabolism, Medical University of Vienna, Wien, Austria

${ }^{2}$ Section for Science of Complex Systems, Medical University of Vienna, Vienna, Austria

${ }^{3}$ Complexity Science Hub Vienna, Vienna, Austria

${ }^{4}$ Department of Psychiatry and Psychotherapy, Division of Social Psychiatry,

Medical University of Vienna, Vienna, Austria

${ }^{5}$ Gender Institute, Gars am Kamp, Austria

Contributors CD wrote the manuscript and researched data. ED researched data, compiled graphs and tables, reviewed/edited the manuscript. ML contributed to the methods and reviewed/edited the manuscript LK-T contributed to the discussion and reviewed the manuscript. PK researched data and reviewed/edited the manuscript. AK contributed to the discussion and reviewed/edited the manuscript AK-W contributed to the discussion and reviewed/edited the manuscript. AK-W is the guarantor of this work and, as such, had full access to all the data in the study and takes responsibility for the integrity of the data and the accuracy of the data analysis.

Funding Funding was received from the WWTF - Vienna Science and Technology Fund (MA16-045)

Competing interests None declared.

Patient consent for publication Not required.

Provenance and peer review Not commissioned; externally peer reviewed.

Data availability statement Data are available on reasonable request. All data relevant to the study are included in the article or uploaded as supplementary information. The analysis is based on secondary use of a research database of medical claims records, which is safeguarded and maintained by the Austrian National Institute for Public Health (GÖG).This is a consolidated research database that is only accessible for selected partners under a strict data protection policy. Use of the data takes place in agreement and cooperation with the GÖG.

Open access This is an open access article distributed in accordance with the Creative Commons Attribution Non Commercial (CC BY-NC 4.0) license, which permits others to distribute, remix, adapt, build upon this work non-commercially, and license their derivative works on different terms, provided the original work is properly cited, appropriate credit is given, any changes made indicated, and the use is non-commercial. See: http://creativecommons.org/licenses/by-nc/4.0/.

ORCID iD

Alexandra Kautzky-Willer http://orcid.org/0000-0002-3520-4105

\section{REFERENCES}

1 World Health Organization. Global status report on noncommunicable diseases 2014 - Global Target 7: Halth the rise in diabetes and obesity, 2014. Available: http://www.who.int/nmh/ publications/ncd-status-report-2014/en/

2 Wild S, Roglic G, Green A, et al. Global prevalence of diabetes: estimates for the year 2000 and projections for 2030. Diabetes Care 2004;27:1047-53.

3 Kautzky-Willer A, Harreiter J, Pacini G. Sex and gender differences in risk, pathophysiology and complications of type 2 diabetes mellitus. Endocr Rev 2016;37:278-316.

4 Naicker K, Johnson JA, Skogen JC, et al. Type 2 diabetes and comorbid symptoms of depression and anxiety: longitudinal associations with mortality risk. Diabetes Care 2017;40:352-8. 
5 Semenkovich K, Brown ME, Svrakic DM, et al. Depression in type 2 diabetes mellitus: prevalence, impact, and treatment. Drugs 2015;75:577-87.

6 Pan A, Lucas M, Sun Q, et al. Bidirectional association between depression and type 2 diabetes mellitus in women. Arch Intern Med 2010;170:1884-91.

7 Egede LE, Zheng D, Simpson K. Comorbid depression is associated with increased health care use and expenditures in individuals with diabetes. Diabetes Care 2002;25:464-70.

8 Frasure-Smith N, Lespérance F, Talajic M. Depression and 18-month prognosis after myocardial infarction. Circulation 1995;91:999-1005.

9 Roy T, Lloyd CE. Epidemiology of depression and diabetes: a systematic review. J Affect Disord 2012;142:S8-21.

10 Barth J, Schumacher M, Herrmann-Lingen C. Depression as a risk factor for mortality in patients with coronary heart disease: a metaanalysis. Psychosom Med 2004;66:802-13.

11 Su C-H, Chiu H-C, Hsieh H-M, et al. Healthcare utilization and expenditures for persons with diabetes comorbid with mental illnesses. Psychiatr Q 2016;87:545-57.

12 Peters SAE, Huxley RR, Woodward M. Diabetes as risk factor for incident coronary heart disease in women compared with men: a systematic review and meta-analysis of 64 cohorts including 858,507 individuals and 28,203 coronary events. Diabetologia 2014;57:1542-51.

13 Wake R, Yoshiyama M. Gender differences in ischemic heart disease. Recent Pat Cardiovasc Drug Discov [Internet] 2009;4:234-40.

14 Soares CN. Depression in peri- and postmenopausal women: prevalence, pathophysiology and pharmacological management. Drugs Aging 2013;30:677-85.

15 Kuehner $\mathrm{C}$. Why is depression more common among women than among men? Lancet Psychiatry 2017;4:146-58.

16 Angst J, Gamma A, Gastpar M, et al. Gender differences in depression. epidemiological findings from the European DEPRES and II studies. Eur Arch Psychiatry Clin Neurosci 2002;252:201-9.

17 Kwong ASF, Manley D, Timpson NJ, et al. Identifying critical points of trajectories of depressive symptoms from childhood to young adulthood. J Youth Adolesc 2019;48:815-27.

18 Nolen-Hoeksema S, Girgus JS. The emergence of gender differences in depression during adolescence. Psychol Bull 1994;115:424-43.

19 Patist CM, Stapelberg NJC, Du Toit EF, et al. The brain-adipocytegut network: linking obesity and depression subtypes. Cogn Affect Behav Neurosci 2018;18:1121-44.

20 Khandaker GM, Zuber V, Rees JMB, et al. Shared mechanisms between coronary heart disease and depression: findings from a large UK general population-based cohort. Mol Psychiatry 2020;25:1477-86

21 Vittengl JR. Mediation of the bidirectional relations between obesity and depression among women. Psychiatry Res 2018;264:254-9.

22 Cohen LS, Soares CN, Vitonis AF, et al. Risk for new onset of depression during the menopausal transition: the Harvard study of moods and cycles. Arch Gen Psychiatry 2006;63:385-90.

23 Bromberger JT, Schott LL, Kravitz HM, et al. Longitudinal change in reproductive hormones and depressive symptoms across the menopausal transition: results from the study of women's health across the nation (Swan). Arch Gen Psychiatry 2010;67:598-607.

24 Freeman EW, Sammel MD, Lin $\mathrm{H}$, et al. Associations of hormones and menopausal status with depressed mood in women with no history of depression. Arch Gen Psychiatry 2006;63:375-82.

25 Sigmon ST, Pells JJ, Boulard NE, et al. Gender differences in SelfReports of depression: the response bias hypothesis revisited. Sex Roles 2005;53:401-11.

26 House J, Marasli P, Lister M, et al. Male views on help-seeking for depression: a Q methodology study. Psychol Psychother 2018;91:117-40.
27 Cavanagh A, Wilson CJ, Kavanagh DJ, et al. Differences in the expression of symptoms in men versus women with depression: a systematic review and meta-analysis. Harv Rev Psychiatry 2017;25:29-38-38.

28 Stapelberg NJC, Neumann DL, Shum DHK, et al. A topographical map of the causal network of mechanisms underlying the relationship between major depressive disorder and coronary heart disease. Aust N Z J Psychiatry 2011;45:351-69.

29 Zhang P, Gao J, Pu C, et al. Apolipoprotein status in type 2 diabetes mellitus and its complications (review). Mol Med Rep 2017;16:9279-86.

30 Palmer S, Vecchio M, Craig JC, et al. Prevalence of depression in chronic kidney disease: systematic review and meta-analysis of observational studies. Kidney Int 2013;84:179-91.

31 Chrysohoou C, Kollia N, Tousoulis D. The link between depression and atherosclerosis through the pathways of inflammation and endothelium dysfunction. Maturitas 2018;109:1-5-5.

32 Robinson RG, Jorge RE. Post-Stroke depression: a review. Am J Psychiatry 2016;173:221-31.

33 Luck-Sikorski C, Schomerus G, Jochum T, et al. Layered stigma? co-occurring depression and obesity in the public eye. J Psychosom Res 2018;106:29-33

34 Erol A, Karpyak VM. Sex and gender-related differences in alcohol use and its consequences: contemporary knowledge and future research considerations. Drug Alcohol Depend 2015;156:1-13.

35 Åhlin J, Hallgren M, Öjehagen A, et al. Adults with mild to moderate depression exhibit more alcohol related problems compared to the general adult population: a cross sectional study. BMC Public Health 2015;15:542.

36 Marmorstein NR. Longitudinal associations between alcohol problems and depressive symptoms: early adolescence through early adulthood. Alcohol Clin Exp Res 2009;33:49-59.

37 Strandheim A, Holmen TL, Coombes L, et al. Alcohol intoxication and mental health among adolescents - a population review of 8983 young people, 13-19 years in North-Trøndelag, Norway: the Young-HUNT study. Child Adolesc Psychiatry Ment Health 2009;3:1-7

38 Fleming CB, Mason WA, Mazza JJ, et al. Latent growth modeling of the relationship between depressive symptoms and substance use during adolescence. Psychol Addict Behav 2008;22:186-97.

39 Boden JM, Fergusson DM. Alcohol and depression. Addiction 2011;106:906-14.

40 Vaccarino V, Goldberg J, Magruder KM, et al. Posttraumatic stress disorder and incidence of type-2 diabetes: a prospective twin study. J Psychiatr Res 2014;56:158-64.

41 Perrin NE, Davies MJ, Robertson N, et al. The prevalence of diabetes-specific emotional distress in people with type 2 diabetes: a systematic review and meta-analysis. Diabet Med 2017;34:1508-20.

42 Wardian JL, Tate J, Folaron I, et al. Who's distressed? A comparison of diabetes-related distress by type of diabetes and medication. Patient Educ Couns 2018;101:1490-5.

43 Peters TM, Pelletier R, Behlouli H, et al. Excess psychosocial burden in women with diabetes and premature acute coronary syndrome. Diabet Med 2017;34:1568-74.

44 O'Connor PJ, Crain AL, Rush WA, et al. Does diabetes double the risk of depression? Ann Fam Med 2009;7:328-35.

45 Branney P, White A. Big boys don't cry: depression and men. Adv. psychiatr. treat 2008;14:256-62.

46 Galdas PM, Cheater F, Marshall P. Men and health help-seeking behaviour: literature review. J Adv Nurs 2005;49:616-23.

47 Thompson AE, Anisimowicz Y, Miedema B, et al. The influence of gender and other patient characteristics on health care-seeking behaviour: a QUALICOPC study. BMC Fam Pract 2016;17:38-7. 